\title{
VII. Zwischen NS-Brutalität, polnischer Diplomatie und deutsch-jüdischem Establishment
}

\section{Die Ausweisung nach Zbanszyn: Eine Generalprobe}

Am 31. März 1938 verabschiedeten beide Kammern des polnischen Parlaments ein Gesetz, das es dem polnischen Innenminister ermöglichte, Personen die polnische Staatsbürgerschaft zu entziehen, die länger als fünf Jahre außerhalb Polens gelebt oder gegen den polnischen Staat agitiert, die ihre Verbindungen mit der polnischen Administration oder dem polnischen Volk abgebrochen oder sich deutlich in einer den Ruf Polens schädigenden Weise verhalten hatten ${ }^{1}$. Weder im Gesetz noch in seiner Begründung werden Juden erwähnt, doch es war allseits bekannt, daß sich das Gesetz gegen die im Deutschen Reich lebenden polnischen Juden richtete ${ }^{2}$. Ausschlaggebende Faktoren für die Verabschiedung dieses Gesetzes waren die Annexion Österreichs durch NS-Deutschland und die daraus resultierende Befürchtung der polnischen Regierung, Tausende von polnischen Juden könnten aus den Gebieten (des ehemaligen) Österreichs nach Polen remigrieren ${ }^{3}$. Die polnischen Motive waren jedoch noch komplexer: Die Aktien der polnischen Raffinerien in Galizien befanden sich überwiegend im Besitz von Juden mit österreichischer Staatsbürgerschaft, die infolge des „Anschlusses“ zu Staatsangehörigen des Deutschen Reiches geworden waren ${ }^{4}$. Die polnische Regierung befürchtete, Deutschland könne den jüdischen Besitz enteignen und so die Kontrolle über die polnischen Raffinerien erlangen. Als vorbeugende Maßnahme schlug der polnische Botschafter in Berlin, Jozef Lipski, vor zu signalisieren, die polnische Regierung wolle sich gegenüber der Enteignung des Besitzes polnisch-jüdischer Bürger in Deutschland „passiv verhalten". Im Gegenzug sollte sich die deutsche Regierung verpflichten, Juden nicht nach Polen auszuweisen und die im Besitz deutscher Staatsangehöriger befindlichen Aktien der polnischen Ölindustrie an Polen zu übergeben. Das polnische Außenministerium beschloß nach Beratungen mit dem Industrie- und Handelsministerium, sich im Hintergrund für die Ausbürgerung polnischer Juden in Deutschland einzusetzen, um so die Bedingungen zur Einziehung ihres Vermögens zu schaffen und die Übertragung der Ölaktien an Polen im Rahmen eines gegenseitigen Vertrages einzuleiten. Die polnische Regierung verschob die Implementierung des Gesetzes

1 Der Gesetzestext in: Wiener Library, Jewish Central Information Office, „Ausbürgerung polnischer Staatsbürger", 613.

2 Dies ergibt sich eindeutig aus einem Gespräch zwischen dem polnischen Botschafter in den Vereinigten Staaten und Vertretern des Jïdischen Weltkongresses: AAN, Amb Rp w Berlinie, Akt Nr. 3896, Jerzy Potocki, Stephan Wise and Louis Lipski, 31.3. 1938 in Washington. Dies wurde auch von der deutschen Botschaft in Warschau so verstanden: Jonca, Niemiecko Polski, S. $108 \mathrm{f}$.

3 Diner, Auswanderung ohne Einwanderung, S. 153.

4 Melzer, No Way out, S. 122. 
auf einen späteren Zeitpunkt und verschaffte sich so zunächst einen Handlungsspielraum. Möglicherweise hoffte sie, in Verhandlungen mit Deutschland über ein Clearing-Abkommen eintreten zu können ${ }^{5}$, vielleicht glaubte sie zunächst noch immer an die Möglichkeit einer beschleunigten polnisch-jüdischen Auswanderung infolge der bevorstehenden Evian-Konferenz ${ }^{6}$. Nachdem jedoch alle diese Erwartungen enttäuscht worden waren, gab der polnische Innenminister am 6. Oktober 1938 eine Anordnung heraus, der zufolge eine Kontrolle und eine einmalige Abstempelung aller polnischen Pässe bis zum 30. Oktober 1938 zu erfolgen hatte. Aufgrund der neuen Anordnung waren ausschließlich Besitzer derartig gestempelter und verlängerter Pässe berechtigt, künftig nach Polen einzureisen. Die polnischen Konsulate in Deutschland weigerten sich jedoch, vorgelegte Pässe abzustempeln. $\mathrm{Nachdem}$ der Intervention des deutschen Außenministeriums beim polnischen $\mathrm{Au}-$ ßenminister, den genannten Termin zurückzunehmen oder sich zu verpflichten, auch in Zukunft polnischen Staatsbürgern ohne abgestempelten Paß die Einreise nach Polen zu erlauben, nicht entsprochen worden war7, wies SS-Führer Heinrich Himmler alle verantwortlichen Stellen an, präventiv und vor Ablauf des polnischen Ultimatums zu handeln; in einer einmaligen Aktion wies Deutschland in der Zeit vom Abend des 27. bis zum 29. Oktober 193817000 polnische und staatenlose Juden polnischer Herkunft aus dem Reichsgebiet aus ${ }^{8}$.

Dieser Schritt der deutschen Seite war nicht ausschließlich eine Reaktion auf die polnische Politik. Im Verlauf der ersten Hälfte des Jahres 1938 hatte Deutschland generell mit kollektiven Ausweisungen ausländischer Juden begonnen. Insbesondere betroffen waren Juden mit russischer und rumänischer Staatsbürgerschaft ${ }^{9}$. Mit Polen jedoch wollte Deutschland Verhandlungen führen, die die wahren Absichten der Nationalsozialisten tarnen sollten: In Gesprächen des polnischen Botschafters in Deutschland, Lipski, mit Hitler und dem deutschen Außenminister, von Ribbentrop, gab die deutsche Seite im Laufe der Monate September und Oktober 1938 ihre Bereitschaft zu verstehen, mit Polen zu kooperieren und geeignete Zielländer für eine jüdische Emigration zu suchen ${ }^{10}$. Tatsächlich handelte es sich dabei um einen Köder, mit dem Polen zu territorialen Verzichten in Danzig und dem polnischen Korridor veranlaßt werden sollte ${ }^{11}$. Noch während der Verhandlungen beschloß Deutschland die Ausweisung von Juden mit polnischer Staatsbürgerschaft eine Mahnung an die polnische Regierung, Deutschland sei durchaus in der Lage, das Problem der jüdischen Bevölkerung in Polen zu verschärfen, wenn Polen sich bei den diplomatischen Verhandlungen mit Deutschland nicht flexibel zeige.

Am Abend des 27. Oktober 1938 wurden mit Hilfe der Polizei aufgrund von Namenslisten 17000 polnische Juden in ganz Deutschland zusammengetrieben und

5 Ebenda, S. 296. Dies ergibt sich auch aus einem Dokument des polnischen Außenministeriums: Akten zur deutschen Außenpolitik, Aide-Memoire des polnischen Außenministeriums am 27. 10. 1938, Dok. 52/34470-471, S. 97.

${ }^{6}$ Diner, Auswanderung ohne Einwanderung, S. 159.

7 Maurer, Abschiebung, S. 59; Akten zur deutschen Außenpolitik, Der Leiter der Rechtsabteilung an die Botschaft in Warschau, 26. 10. 1938, Dok. 52/34469, S. 93.

8 Maurer, Abschiebung; Milton, Expulsion; dies., Grenzen; Adler, Der verwaltete Mensch, Kap. 4; Bauer, Brother's Keeper, S. 243-252; Goldberg, Zwangsausweisung.

${ }^{9}$ Milton, Grenzen, S. $189 \mathrm{ff}$.

10 Lipski, Diplomat in Berlin, S. $411 \mathrm{f} ., 458$.

11 Melzer, Polish Diplomacy, S. 229. 
im Laufe von zwei Tagen über die polnische Grenze gebracht. Die Anordnungen zur Verhaftung der polnischen Juden und zur Ausführung der Aktion waren von Ort zu Ort verschieden: in großen Städten waren hauptsächlich Männer über 16 Jahre betroffen, in kleineren Ortschaften die gesamte jüdisch-polnische Bevölkerung. In den meisten Fällen wurde es den Betroffenen nur gestattet, wenig Gepäck und pro Person zehn Mark mitzunehmen. Bisweilen konnten sie persönliche Gegenstände und höhere Geldsummen mit sich führen. Sie wurden mit der Eisenbahn in die Nähe der polnischen Grenze gebracht und unter Drohung mit Gewehren über die grüne Grenze getrieben - ins Niemandsland zwischen den Staaten. Die polnischen Grenzposten reagierten unterschiedlich: manchmal gestatteten sie den Ausgewiesenen die Einreise nach Polen, manchmal nicht. Ein Teil der Menschen gelangte so auf polnisches Gebiet, andere verblieben im Niemandsland, einige kehrten nach Deutschland zurück, nachdem der Ausweisungsversuch auf Schwierigkeiten gestoßen war. In diesem Zusammenhang wurde insbesondere die polnische Grenzstadt Zbanszyn bekannt, in der sich 6000 jüdisch-polnische Ausgewiesene konzentrierten, denen die Einreise nach Polen verweigert worden war und die dort einige Monate unter schwersten Bedingungen zubringen mußten, während derer ihr Schicksal die Aufmerksamkeit der jüdischen Öffentlichkeit in aller Welt auf sich lenkte.

Die Ausweisungen wurden erst eingestellt, nachdem Polen seinerseits als Reaktion mit der Ausweisung deutscher Staatsbürger begonnen hatte. Am 2. November 1938 wurden dann Verhandlungen zwischen beiden Staaten über das Schicksal der Flüchtlinge aufgenommen, die durch das Attentat auf den deutschen Gesandtschaftsrat in Paris unterbrochen wurden. Ausgeübt wurde das Attentat von Herschel Grynszpan, dessen Eltern zu den Ausgewiesenen in Zbanszyn gehörten. Als Antwort auf dieses Attentat organisierten die NS-Behörden einen landesweiten Pogrom, die sogenannte „Reichskristallnacht“. Am Ende der Verhandlungen verpflichtete sich Polen aufgrund eines am 24. Januar 1939 unterzeichneten Vertrages, die Zusammenführung von in Deutschland lebenden Familienangehörigen mit ihren Verwandten in Polen zu gestatten. Im Gegenzug ermöglichte Deutschland den Ausgewiesenen die Rückkehr für einen auf acht Monate begrenzten Zeitraum, damit die Betroffenen ihre finanziellen Angelegenheiten regeln und ihre Unternehmen in Deutschland auflösen konnten. Bedingung war allerdings, daß jeweils nicht mehr als tausend Juden zurückkehrten. Außerdem sollte diese Aktion bis Ende Juni 1939 abgeschlossen werden. Deutschland verpflichtete sich, den Ausgewiesenen die Mitnahme ihres persönlichen Besitzes sowie der für ihre Berufsausübung notwendigen Gerätschaften zu gestatten. Beide Staaten kamen überein, ein gemeinsames Konto für die Auflösung des zurückgebliebenen Besitzes der so aus Deutschland Vertriebenen einzurichten. Trotz dieser Verpflichtungen setzte Deutschland während jener Monate die Ausweisung polnischer Juden aus seinem Gebiet, wenn auch in geringerem Umfange, fort.

Das Verhalten des nationalsozialistischen Deutschlands den polnischen Juden gegenüber erklärt sich aus dem Gesamtkomplex der Außenbeziehungen des Reiches, insbesondere zum polnischen Nachbarn. Die Annexion Österreichs im März 1938 und die Kapitulation Frankreichs und Großbritanniens in der Frage des Sudetenlandes im September 1938 hatten der deutschen Außenpolitik zweifellos wichtige Signale gegeben. Die deutsche Regierung hatte verstanden, daß ihr außenpolitisch 
kein Hindernis mehr in den Weg gelegt wurde. Das Zögern, das die auf der EvianKonferenz vertretenen Länder im Hinblick auf eine Lösung der Frage der jüdischen Flüchtlinge aus Österreich und Deutschland zeigten, verstärkte den Eindruck, es existiere keine internationale aktive Lobby, die sich für den Schutz von Juden oder ihre Emigration einsetze. Die durch eine aggressive Emigrationspolitik herbeigeführten „Erfolge“ in Wien galten als schlagender Beweis für die eingesetzte Methode. Vor diesem Hintergrund also war es Ende 1938 deutlich, daß Deutschland die Absicht des polnischen Staates, Zehntausende von in Deutschland lebenden polnischen Juden auszubürgern und ihnen damit die Möglichkeit einer Rückkehr nach Polen oder der Emigration in andere Länder zu nehmen, nicht tatenlos hinnehmen würde.

Das einziges Hindernis im nahezu unbegrenzten Handlungsspielraum des Deutschen Reiches waren allerdings Schicksal und Status der deutschen Minderheit in Polen. Der notwendige Schutz dieser Minderheit war ein Pfand für den Schutz des Status von polnischen Juden und Christen in Deutschland. So reagierte Polen, wie erwähnt, zwei Tage nach der Ausweisung von polnischen Juden aus Deutschland mit der Deportation deutscher Bürger, zum Teil jüdischer Herkunft, aus den polnischen Gebieten Posens und Pommerns ${ }^{12}$.

Deutschland hatte bei der Ausweisungsaktion, zu der es nicht zuletzt durch die irreführenden Erwägungen polnischer Diplomatie gebracht worden war, einen maximalen Erfolg erzielt. Mit dem im Januar 1939 unterzeichneten deutsch-polnischen Abkommen hatte Deutschland zunächst alle seine Ziele erreicht. Ob die Vereinbarungen jedoch auch für die polnische Seite effektiv und sinnvoll waren, muß angezweifelt werden ${ }^{13}$. Ein bedeutender Teil des Besitzes, den die polnischen Juden in der ihnen in Deutschland zum Zwecke der Regelung und Auflösung ihrer Angelegenheiten zugestandenen Zeit liquidieren sollten, wurde angesichts bürokratischer Schwierigkeiten und im Kontext der „Arisierung" unter Zeitdruck und unter Wert veräußert. Die auf gemeinsamen deutsch-polnischen Konten eingezahlten Gewinne verblieben nach Kriegsausbruch in Deutschland. Auf diese Weise gelang es

12 Akten zur deutschen Außenpolitik, Der Reichsführer SS und Chef der Deutschen Polizei an den Chef der Reichskanzlei am 29. 10. 1938, Dok. Nr. 1522/373329-332, S. 98; Jonca, Niemiecko Polski, S. 114 f.; Amb Rp w Berlinie, Akt Nr. 875, Verbalnote des Auswärtigen Amtes Berlin an die polnische Botschaft am 22. 12. 1938. - Einen ähnlichen Schritt hatte Polen 1923 in Reaktion auf die Ausweisung polnischer Juden aus Bayern ergriffen; Maurer, Abschiebung, S. 55.

13 Adler erkennt in dem Abkommen zwischen Deutschland und Polen die deutsche Bereitschaft, seine Politik gegenüber Juden - selbst zu einem so späten Zeitpunkt und nach der Reichspogromnacht - infolge der Intervention ausländischer Kräfte oder Regierungen zu mäßigen. Dies ist m.E. eine irrtümliche Interpretation des Ablommens, das praktisch sehr wohl den deutschen Interessen entgegenkam und sicherlich als cin Erfolg der deutschen Außenpolitik, keineswegs aber als Kapitulation vor externem Druck verstanden werden darf; Adler, Der verwaltete Mensch, S. 100. - Eine differenziertere Variante des gleichen Arguments bringt Maurer, Attentat, S. 72, die davon ausgeht, im Kontext der Ausweisungen polnischer Juden erweise sich, daß die deutsche Außenpolitik 1938 noch immer im Rahmen internationaler Sachzwänge handelte und bei der Ausweisung ausländischer Juden eher zögerte und auf äußere Umstände wartete, die eine Ausweisung als entsprechende Reaktion ermöglichten. 
Deutschland, die Vertreibung der Juden geordnet, effektiv und gewinnbringend durchzuführen ${ }^{14}$.

Die Ausweisung nach Polen war die erste einer Reihe mehrerer kollektiver Ausweisungen, die die Nationalsozialisten in der Folge durchführen sollten. Sie erweist sich aus dem Rückblick als eine Art „Generalprobe“15. Sybil Milton geht davon aus, daß es sich um „den ersten Versuch in der Organisatinion technischer Details für spätere Ausweisungen nach 1941" handelte ${ }^{16}$. Die Ausweisung nach Polen war auf jeden Fall ein Akt, an dem die verantwortlichen NS-Stellen im Laufe der Ereignisse des Oktobers 1938 erproben konnten, was sie bei späteren Vertreibungen einsetzten, auch wenn sie zu diesem Zeitpunkt noch keine weiteren, ähnlichen Aktionen bewußt planten.

Eine der wichtigsten Lehren, die aus der „Polenaktion“ gezogen werden konnten, betraf die Arbeit der deutschen Polizei, die sich in hohem Maße als ungeeignet für die Durchführung derartiger Maßnahmen erwiesen hatte. Selbst die Betroffenen spürten eine gewisse Zurückhaltung und Rücksicht der deutschen Polizisten bei der Begegnung mit den Ausgewiesenen ${ }^{17}$, obwohl ihnen schwere Sanktionen für jede Rücksichtsnahme oder Sympathie drohten:

„Auf den Stationen, wo wir hielten, baten wir die begleitenden Polizeibeamten, uns Milch für die kleinen Kinder zu kaufen. Sie weigerten sich, da sie strengen Befehl hatten, dies nicht zu tun. Als sich aber ein Polizeibeamter doch erweichen ließ und für ein krankes kleines Kind Milch brachte, durfte keiner der begleitenden Polizeibeamten den Bahnsteig an den Haltepunkten mehr betreten. Sie mußten in ihren Abteilen sitzen bleiben und einsam patroullierte der Offizier auf und ab ..."18

Einer der Betroffenen berichtete seiner Schwester über eine gewisse Annährung zwischen Polizisten und Ausgewiesenen, ja sogar über humorvolle Gespräche, die er zu schätzen wußte, nachdem er das Verhalten der SS-Männer - von ihm als „Verbrecher" bezeichnet - kennengelernt hatte ${ }^{19}$. Auch in Leipzig zeigte die örtliche Polizei eine gewisse Flexibilität, indem sie es der Gemeinde ermöglichte, Personen freizubekommen, die mit Hilfe medizinischer Gutachten eines älteren jüdischen Sanitäters als nicht reisefähig erklärt wurden ${ }^{20}$. Aus anderen Berichten ergibt sich, daß

14 Deutsch-polnische Verhandlungsdokumente: AAN, Amb Rp w Berlinie, Akt 883; Beispiele für die Auflösung polnisch-jüdischen Besitzes in Deutschland: StA Leipzig, Devisen-Stelle Leipzig 185; StA Hamburg, Oberfinanzpräsident 314-315, Sign: 42 U.A. 9, F-Vg 7651, 2071/39.

15 Dieser Ausdruck wird von Hans Pomeranz, einem jüdischen Aktivisten der Gemeinde in Frankfurt, in seiner Aussage benutzt: YVA, 01/275, Dr. Hans Pomeranz. - Zur gleichen Zeit wurden Juden mit ungeklärter Staatsangehörigkeit aus dem Sudetenland, aus Ungarn und aus der Slowakei ausgewiesen. Sie wurden in Flüchtlingslagern im Grenzland untergebracht; Milton, Expulsion, S. 169.

16 Milton, Grenzen; dies., Expulsion, S. 171-174; Maurer, Abschiebung, S. 71.

17 Maurer, Abschiebung, S. 63.

18 YVA, M-1/E-1903/1753, Josef Kohns, Leiter der Jewish Historical Commission, 28. 10. 1938.

19 Brief Julius Rosenzweigs (Warschau) an seine jüngere Schwester Celia vom 19. 8. 1939; nach der Übersetzung bei Milton, Expulsion, S. 177f. Der Verfasser bemerkt u.a., als der Transport in der Kleinstadt Beuthen hielt und die jüdische Ortsgemeinde Nahrungsmittel und Getränke zur Verfügung stellte, hätten die deutschen Polizisten mit den Ausgewiesenen zusammen gegessen.

20 StA Dresden, Ministerium des Innern, Nr. 11180, Der Polizeipräsident zu Leipzig an den Kreishauptmann zu Leipzig am 26. 11. 1938. Nach zuverlässigen Aussagen fragte der Poli- 
weniger disziplinierte deutsche Polizisten das Geld der Ausgewiesenen beschlagnahmten und in die eigene Tasche steckten ${ }^{21}$. Aus Oberschlesien wurde berichtet, daß die Ausweisung unter gemeinsamer Kontrolle von SS und SA durchgeführt wurde, da sich auch die SA als unzuverlässig erwiesen und Raub an den Ausgewiesenen nicht verhindert hatte ${ }^{22}$.

In Leipzig kam es zu schweren Auseinandersetzungen zwischen der Gestapo und der SS sowie der örtlichen Polizei. Schon am Nachmittag des 27. Oktober 1938 hatte die Leipziger Polizei die Anweisung erhalten, polnische Juden aus der Stadt auszuweisen. Infolge des Legalismus der Polizeiführung verzögerte sich die Ausweisung um einige Stunden und wurde erst für den Morgen des 28. Oktober angesetzt ${ }^{23}$. Diese Verzögerung hätte keine weiteren Auswirkungen gehabt, wenn der polnische Konsul in Leipzig nicht in der Zwischenzeit Informationen über die in Gang befindliche Deportationsaktion erhalten hätte. Er rief die Juden Leipzigs auf, ihre Dokumente zu verbrennen und sich auf dem Konsulatsgelände zu versammeln, das er ihnen öffnete. Hunderte von Juden kamen zum Konsulat und fanden auf diesem polnischen Territorium Schutz und Zuflucht. Die Gestapo reagierte mit heftiger Kritik an der mangelnden Funktionstüchtigkeit der lokalen Polizei und der Unfähigkeit des lokalen Polizeikommandanten, derartige Sonderaktionen durchzuführen. Die SS bewies hingegen bei der „Polenaktion“ äußerste Effizienz, Entschlossenheit und Grausamkeit gegenüber den Ausgewiesenen. Dieses Verhalten unterstrich ohne Zweifel ihr Image als eine Organisation, die sich der Zivilbevölkerung gegenüber ohne jegliche Rücksichten verhielt. Es festigte die Position der SS bei späteren Vertreibungs- und Mordaktionen ${ }^{24}$.

Die Ausweisung polnischer Juden aus Deutschland Ende 1938 war darüber hinaus der erste Fall einer kollektiven Massenausweisung aus Deutschland unter dem NS-Regime, der es den Regierungsstellen ermöglichte, das gesamte Spektrum möglicher Reaktionen der deutschen Öffentlichkeit zu testen, da er sich in der Öffentlichkeit abspielte und nicht im Verborgenen. Die Ausgewiesenen wurden auf den Straßen aufgegriffen oder aus Wohnhäusern herausgeholt, sie wurden durch die Straßen geführt und bestiegen auf den von Menschen belebten Bahnhöfen die Eisenbahnwaggons. Ein Ausgewiesener aus Chemnitz beschrieb seinen Weg zur Eisenbahn:

"Als wir hinaus auf die Straße kamen, regnete es fürchterlich. Im Treppenhaus und auf der Straße standen Menschen herum und guckten, andere schauten aus ihren Fenstern. Wir wurden auf Polizeiautos verfrachtet und eiligst zum Bahnhof gefahren. Auch hier standen Menschen und sahen alles, aber es waren auch Juden da, die sich verabschieden wollten." 25

zeihauptmann das Wohlfahrtsamt der jüdischen Gemeinde in Leipzig, ob sie alle Ausgewiesenen freistellen könnten, nahm jedoch nicht aktiv an der Wahl der Betroffenen teil: YVA, 01/147, Martin Altertum, Das Socialamt der Jüdischen Gemeinde in Leipzig.

$21 \mathrm{SoPaDe}$, November, Berlin, 1185-86.

22 SoPaDe, Oberschlesien, November 1938, A 37, 1196.

23 Zum Verlauf der Ereignisse in Leipzig aus der Perspektive der Polizei: StA Dresden, Ministerium des Innern, Nr. 11180, Polizeipräsident zu Leipzig an den Kreishauptmann zu Leipzig am 26. 11. 1938; Geheime Staatspolizei (Dresden) an den Reichsführer SS und Chef der Deutschen Polizei im Reichsministerium des Innern am 9. 11. 1938.

24 Diner, Auswanderung ohne Einwanderung, S. 160.

25 Julius Rosenzweig bei: Milton, Expulsion, S. 177. 
Angesichts der allgemeinen Indifferenz der deutschen Öffentlichkeit gegenüber der antijüdischen NS-Politik im Jahre $1938^{26}$ gab es keinerlei Grund, empathische Gefühle für Juden oder gar Widerstand gegen die Ausweisungen unter der deutschen Bevölkerung zu fürchten ${ }^{27}$. Dennoch versuchten die Behörden die Aktion so weit wie möglich im Verborgenen durchzuführen. Den Ausgewiesenen wurde befohlen, die Fenster der Eisenbahnwaggons zu schließen oder ins Innere der Wagen zurückzutreten. Oft wurden sie mit Waffengewalt von den Fenstern ferngehalten. In manchen Fällen waren die Waggons versiegelt ${ }^{28}$.

Während sich die deutsche Gesellschaft gegenüber den Ausgewiesenen weitgehend gleichgültig verhielt, mußten sich die Behörden mit eventuellen wirtschaftlichen Konsequenzen der Ausweisung für deutsche Bürger auseinandersetzen ${ }^{29}$. Die überstürzten Ausweisungen verursachten verschiedene Probleme. Viele der Ausgewiesenen waren nicht in der Lage, ihre finanziellen Außenstände bei Angestellten, Vermietern oder anderen Privatpersonen zu begleichen. Bisweilen kam es zu Übergriffen, bei denen Angehörige der Deutschen Arbeitsfront sich des Besitzes der Ausgewiesenen mit dem Argument bemächtigten, sie würden die den Arbeitern zustehenden Außenstände eintreiben ${ }^{30}$. Auch mußte die Frage der Verfügung über die Wohnungen der Ausgewiesenen beantwortet werden, und zwar sowohl im Hinblick auf die Vermieter als auch die vielen Wohnungssuchenden, die sich für die verlassenen Wohnungen zu interessieren begannen. Ebenso mußte über den in den Wohnungen verbliebenen Besitz entschieden werden. In Sachsen $z$. B. gaben die Gerichte dem Druck nach und stellten die Wohnungen der Ausgewiesenen für eine Versteigerung frei ${ }^{31}$, zum Mißfallen des deutschen Auswärtigen Amts, das Schadensersatzforderungen befürchtete. Hier kam es zu Interventionen der jüdischen Gemeinden.

Aus den Aussagen zahlreicher Ausgewiesener ergibt sich, daß die jüdischen Gemeinden den Betroffenen an jedem Ort hilfreich zur Seite standen. Dieser Hilfsbereitschaft kommt in den Erinnerungen der Betroffenen große Bedeutung zu, weil sie der einzige Lichtblick in dem Alptraum der Ausweisung war und wohl auch, weil dieses Verhalten im krassen Gegensatz zu einer gewissen Distanz stand, die viele der osteuropäischen Juden in Deutschland bis dahin seitens der altansässigen Juden ihnen gegenüber verspürt hatten. Ein zionistischer Aktivist aus Frankfurt berichtete, zur Stunde der Ausweisung aus Frankfurt seien alle Gegensätze und gesellschaftlichen Unterschiede zwischen ansässigen und ausgewiesenen Juden fortgefallen: „Auch die assimilierten Kreise, die sich damals insbesondere um den Reichsbund jüdischer Frontsoldaten scharten, verstanden, daß es sich hierbei um eine Ge-

26 Siehe dazu: Bankier, Öffentliche Meinung, S. $113 \mathrm{ff}$.

27 Dr. Eschelbacher, der die Vertreibung von Juden aus Düsseldorf beobachtete, beschrieb die Reaktion der Straße mit den Worten: „Die Menge auf der Straße war totenstill, aber gelegentlich hörte man doch boshafte und hämische Bemerkungen." - YVA, 02/23.

28 YVA, 02/427, Die „Polenaktion“; 02/486, Brief eines im Zuge der „Polenaktion“ deportierten Berliner Juden, Krakau, 1. 11. 1938; 01/170, Brief von Mary Sprei, 14. 11. 1938, Krakau.

29 StA Dresden, Ministerium des Innern Nr. 11180, Reichshauptmann zu Leipzig an den Sächsischen Minister des Innern zu Dresden, 29. 10. 1938.

30 AAN, Amb Rp w Berlinie, Akt Nr. 875, Unter Bezugnahme auf die Verbalnote Nr. N51a/365.

31 StA Dresden, Ministerium des Innern Nr. 11180, Sächsischer Minister des Innern an Reichsführer SS und Chef der Deutschen Polizei, 2. 12. 1938. 
neralprobe handelte."32 Ein Ausgewiesener sah den Grund der Hilfsbereitschaft ebenfalls in der Erkenntnis, daß es sich um „ein Vorspiel für das ihnen (den deutschen Juden) drohende Schicksal" handele, und nicht in der Aufhebung der Spannungen und Gegensätze zwischen west- und osteuropäischen Juden, die seiner Ansicht nach auch in Deutschland keineswegs verschwunden waren ${ }^{33}$. Und eine betroffene Frau schrieb, man müsse zwei Punkte hinsichtlich der seit Generationen in Deutschland verwurzelten Juden hervorheben: Sie hätten der osteuropäisch-jüdischen Gemeinde gegenüber stets eine gewisse Distanz gezeigt. In der Stunde der Not jedoch seien sie über ihren Schatten gesprungen und hätten ihren bedürftigen Glaubensbrüdern geholfen, sie hätten den Internierten Lebensmittel gebracht und für Rat und Trost gesorgt ${ }^{34}$. Dieses Verhalten war also nicht das Ergebnis eines langsamen Prozesses des Abbaus der trennenden Grenzen. Vielmehr war ein dramatisches Ereignis notwendig gewesen, um die Gemeinden aufzurütteln und derartig starke Reaktionen von Sympathie, Mitleid und Unterstützung hervorzurufen, deren Wurzeln in dem Wissen lagen, auch ihnen, den deutschen Juden, könnte in Zukunft ein ähnliches Schicksal drohen.

Die Ausgewiesenen wurden aus ihren Wohnungen geholt, ohne daß sie Gelegenheit hatten, sich auf die Reise vorzubereiten. Anschließend wurden sie stundenlang an Sammelpunkten in den Städten festgehalten. Daher ergab sich dringend die Notwendigkeit, sie schnell mit Lebensmitteln zu versorgen. Viele Gemeinden verpflegten die Ausgewiesenen, erwarben das Notwendigste mit Geld der Gemeindekassen und stellten Lebensmittel für die weitere Fahrt zur Verfügung ${ }^{35}$. Die Gemeinden verteilten auch Bargeld unter den Ausgewiesenen, die in vielen Fällen keinen Pfennig in der Tasche hatten ${ }^{36}$. Oft holte die Gemeinde Ärzte herbei, die Gebrechlichen und Schwachen Reiseunfähigkeit attestieren sollten ${ }^{37}$. Manche Polizeipräsidenten hielten es für richtig, die Hilfe der jüdischen Gemeinden für die Ausgewiesenen in ihren Berichten an vorgesetzte Dienststellen hervorzuheben ${ }^{38}$, eine Hilfe, die, so ergibt sich aus den Berichten der Leipziger Polizei, im Rahmen der ordnungsgemäßen Abwicklung der Ausweisungen gewährt wurde ${ }^{39}$. Darüber hinaus übernahmen viele Gemeinden die Schulden der abgeschobenen Personen, ordneten deren Besitz und Geschäfte und kümmerten sich um die Wohnungen. Es ist relativ schwierig, die Abläufe im einzelnen zu rekonstruieren; es hat jedoch den Anschein, als ob die zu-

32 YVA, 01/275, Dr. Hans Pomeranz.

33 YVA, 02/546, Erich Cohn, Before and after the November Pogrom 1938, (Delft, Holland), 22. 11.1938.

34 YVA, 08/19, Aussage Ellen Trotzki.

35 StA Hamburg, Handschriftensammlung, 1374, Die Situation der jüdischen Bevölkerung Hamburgs im Dritten Reich, Interview von Christel Riecke mit Dr. Max Plaut, März 1973; YVA, 02/23, Rabbiner Dr. Eschelbacher, Das Pogrom vom 10. November 1938 in Düsseldorf.

36 YVA, 01/147, Martin Altertum, Das Socialamt der Jüdischen Gemeinde in Leipzig. Viele betrachteten diese Geldverteilung spöttisch: „Zum Geldnehmen aber meldete sich keiner, was konnte uns Geld in solcher Lage schon helfen?" - YVA, M-1/E-1903/1753, Josef Kohns, Leiter der Jewish Historical Commission, 28. 10. 1938.

37 YVA, 01/147, Martin Altertum, Das Socialamt der Jüdischen Gemeinde in Leipzig.

38 StA Dresden, Ministerium des Innern Nr. 11180, Der Polizeipräsident zu Chemnitz an den Sächsischen Minister des Innern, 29. 10. 1938.

39 StA Dresden, Ministerium des Innern Nr. 11180, Der Polizeipräsident zu Leipzig an den Sächsischen Minister des Innern, 29. 10. 1938. 
nächst spontan gezeigte Bereitschaft der Gemeinden, die Besitzverhältnisse der Ausgewiesenen zu ordnen, von den NS-Behörden bald in eine Pflicht umgemünzt wurde ${ }^{40}$. Max Plaut von der Führung der jüdischen Gemeinde in Hamburg berichtet in seinen Erinnerungen, er habe damals an dem Sammelpunkt in Hamburg von den Betroffenen Vollmachten für deren Besitz erhalten ${ }^{41}$. Der ehemalige Rabbiner der Düsseldorfer Gemeinde, Dr. Eschelbacher, berichtet ebenfalls, die Ausgewiesenen hätten sich an ihn gewandt. Sie „sprachen von ihren Wohnungen, ihren Außenständen, ihren Schulden, von den Sachen, die ihnen zur Reparatur übergeben waren, von Verwandten im Ausland, die um ein Affidavit angegangen, von Verwandten in Polen, die benachrichtigt werden sollten, sie gaben Aufträge für den polnischen Konsul“. Gleichzeitig aber erhielt er von der Düsseldorfer Polizei „die Liste der Abgeschobenen, sodaß wir uns um die Wohnungen kümmern konnten. Es mußte Vorsorge getroffen werden für den Fall, daß sie gekündigt wurden, die Schlüssel mußten verwahrt werden." Im Verlauf der Ausweisungsaktion, insbesondere jedoch danach, mußten sich die jüdischen Gemeinden um den Besitz der Ausgewiesenen kümmern ${ }^{42}$. Als nach der Unterzeichnung des Abkommens zwischen Polen und Deutschland die ersten Juden für kurze Zeit nach Deutschland zurückkamen, um ihre Angelegenheiten zu ordnen, übernahmen die jüdischen Gemeinden auch die Reisekosten innerhalb Deutschlands ${ }^{43}$. Es war eine unerhörte Belastung der ohnehin nahezu leeren Kassen der Gemeinden, die seit langem des Rechtes beraubt waren, Gemeindesteuern einzuziehen ${ }^{44}$. Die deutschen Behörden machten jedoch keinerlei Anstalten, die Gemeinden von dieser Belastung zu befreien ${ }^{45}$.

Die jüdischen Gemeinden in Deutschland spielten also eine zentrale Rolle während der Ausweisung und in den Wochen danach. Sie halfen aus dem Gefühl einer moralischen Verpflichtung gegenüber den ausgewiesenen Mitgliedern der Gemeinde. Die Möglichkeit eines aktiven jüdischen Widerstandes wurde nicht erwogen. Sie entbehrte jeglicher Grundlage. Gleichzeitig konnten sich die deutschen Behörden überzeugen, daß angesichts des fehlenden Widerstands der Ausgewiesenen entsprechende Aktionen in Deutschland mit einem Minimum an Polizeikontrolle

40 Adler, Der verwaltete Mensch, S. 94; Maurer, Abschiebung, S. 64.

41 StA Hamburg, Die Situation der jüdischen Bevölkerung Hamburgs im Dritten Reich, Interview von Christel Riecke mit Dr. Max Plaut, März 1973.

42 Dies ergibt sich aus der Darstellung der Arbeit des jüdischen Wohlfahrtsamtes in Frankfurt: Dokumente zur Geschichte der Frankfurter Juden, zitiert bei: Adler, Der verwaltete Mensch, S. 97. Auch die Berliner Gemeinde übernahm die Kosten für die Rückführung eines Teils der Ausgewiesenen sowie die Kosten für die Rückkehr in Wohnungen und Häuser. YVA, 02/546, Erich Cohn, Before and after the November Pogrom 1938 (Delft, Holland, 22. 11. 1938).

43 CAHJP, Inv. 6528 (207), Gemeinde Darmstadt.

44 Interessant ist die Einschätzung der Situation durch die Gestapo in Dresden-Bautzen: „Die Israelit. Rel. Gem. andererseits kann auf keinen Fall die Kosten für Unterhalt und für Rückfahrt übernehmen. Die polnischen Juden waren die schlechtesten jüdischen Gemeindesteuerzahler, niemand hat sie zurückgerufen und eine [...] ihrer Wünsche wäre für die jüdische Gemeinde [eine] untragbare Belastung. " - StA Dresden, Ministerium des Innern, Nr. 11180, Sicherheitsdienst des Reichsführer SS, SD-Dresden-Bautzen, 5. 5. 1939.

45 Brief des SS-Obergruppenführers Bestof an den Reichsführer SS und Chef der Deutschen Polizei, Berlin, 8. 7. 1939, zitiert bei: Milton, Expulsion, S. 198. 
durchzuführen waren ${ }^{46}$. Die spontane und effektive Hilfsbereitschaft der Mitglieder jüdischer Gemeinden, das einzig denkbare Verhalten angesichts der gegebenen Umstände, unterstützte tragischerweise den Apparat des NS-Regimes und zeigte, in wie zynischer Weise jüdische Solidarität ausgenutzt wurde, um Maßnahmen gegen Juden effektiv und mit maximalem Ergebnis durchzuführen.

\section{Die Ausgewiesenen in Polen: Ein Fehlschlag polnischer Diplomatie}

Die aus Deutschland ausgewiesenen Juden waren ihrer Herkunft bzw. in der Regel ihrer Staatsbürgerschaft nach Polen, doch viele von ihnen beherrschten die polnische Sprache nicht. Teilweise waren sie noch nie in Polen gewesen, oder ihre Sitten und Gebräuche waren - nach jahrzehntelangem Aufenthalt in Deutschland - den polnischen entfremdet. Sie waren Fremde, „Spielball zwischen den Völkern und gleichzeitig Schachfiguren, leblos im Kontext des Politik genannten Spiels" ${ }^{47}$. Sie kamen in ein Land, das seit Jahren eine ausdrücklich antisemitische Politik betrieb, die von breiten Schichten des Volkes unterstützt wurde. Die Ausweisung selbst wurde von Deutschen durchgeführt, sie war jedoch auch eine konsequente Folge der antijüdischen Politik Polens. Trotzdem, entgegen aller Erwartung, nahm die polnische Bevölkerung die Abgeschobenen äußerst freundlich auf ${ }^{48}$. Dieser Umstand, den die Forschung bislang kaum zur Kenntnis genommen hat, zeigt sich in vielen Berichten.

Zirka 4000 Juden überschritten in der Region Beuthen die Grenze nach Polen und gelangten nach Kattowitz, einer Stadt, die in der Vergangenheit geradezu zum Inbegriff eines gewalttätigen Antisemitismus geworden war ${ }^{49}$. Statt auf Feindseligkeit trafen die Ausgewiesenen dort jedoch auf menschliche Zuneigung. Der Leiter des Joint in Polen, Giterman, war von den Sympathiekundgebungen der polnischen Bevölkerung beeindruckt und fühlte sich dadurch in seinem positiven Menschbild bestärkt:

„Dies ist ein weiteres Zeichen dafür, daß dort, wo Haß nicht künstlich gefördert wird, die spontane Reaktion gegen Brutalität durch und durch menschlich ist. Im antisemitischen Kattowitz, wo sich Tausende von Flüchtlingen in den Straßen und Bahnhöfen fanden, habe ich ... nicht das kleinste Anzeichen von Antisemitismus bemerken können. Es ist bemerkenswert, daß einige Christen den Flüchtlingen sogar Geschenke überreichten."

In der Stadt Zbanszyn, in der sich in den ersten Wochen mehr als 6000 Flüchtlinge aufhielten, zeigte sich die einheimische Bevölkerung ebenfalls spontan hilfsbereit, stellte Zimmer zur Verfügung und half bei der Versorgung der Kinder ${ }^{51}$. Das Phänomen überraschte und beschäftigte die Leiter des Joint, die die Hilfsaktionen in

46 Darauf weist Abraham Margaliot, der im Alter von 16 Jahren aus Hamburg ausgewiesen wurde, in seiner Aussage hin: YVA, 03/9/15.

47 Jüdisches Gemeindeblatt Danzig, Nr. 7, 4. 11. 1938.

48 Melzer, No Way Out, S. 124.

49 Milton, Expulsion, S. 171.

50 JDC, Folder 878, Jews Exiled from Germany to Poland, December 1938.

51 JDC, Folder 878, Bericht von Herrn Hellman, 10. 11. 1938. 
Zbanszyn koordinierten. Sie suchten in ihrer grenzenlosen Überraschung nach Erklärungen für die polnische Hilfsbereitschaft ${ }^{52}$. Manche sahen die Ursache im urbanen Erscheinungsbild und Verhalten der Ausgewiesenen. „Diese Flüchtlinge“, so hieß es in einem Bericht, „kamen aus den großen Städten Deutschlands, zum Beispiel aus Berlin, Hamburg, Hannover, Köln, Düsseldorf usw.“ Ein anderer Bericht, der einige Monate nach der Ausweisungsaktion erstellt worden war, hob den wirtschaftlichen Gewinn hervor, den die großstädtischen Flüchtlinge für die unterentwickelte Ortschaft Zbanszyn gebracht hätten ${ }^{53}$. Vielleicht war es der besondere Charakter der Ausgewiesenen, das Fremde, das viele von ihnen in ihrer Kleidung, ihrer Sprache und ihrem Verhalten vermittelten, das sich von dem in Polen üblichen Bild der jüdischen Bevölkerung unterschied und sie vor antisemitischen Ausschreitungen schützte. Die Ausgewiesenen verfügten über eine nicht geringe Kaufkraft, von der die einheimische Bevölkerung profitierte. Auch dies trug dazu bei, daß sie mit Wohlwollen aufgenommen wurden. Darüber hinaus war die Atmosphäre des in Polen am Vorabend des Zweiten Weltkrieges - also gegen Ende 1938, Anfang 1939 herrschenden Antisemitismus sowohl in Regierungskreisen als auch in der Bevölkerung in eine antideutsche Haltung umgeschlagen. Die Ausgewiesenen wurden daher vor allem als Opfer der deutschen Gewalt betrachtet ${ }^{54}$.

Wie reagierte der polnische Staat auf die Situation, zu deren Entstehung er wesentlich beigetragen hatte? 4000 Juden, die die Grenze zwischen Beuthen und Kattowitz überschritten hatten, erhielten vom Gouverneur des Bezirks Schlesien die Genehmigung - und Unterstützung -, Schlesien zu verlassen und ins Innere Polens weiterzuziehen ${ }^{55}$. Auch von den kleinen Grenzübergängen zogen jüdische Flüchtlinge ins Landesinnere. An anderen Orten wurde dem Flüchtlingsstrom Einhalt geboten, so daß sich die Ausgewiesenen in den Grenzorten konzentrierten ${ }^{56}$. Der wichtigste davon war Zbanszyn. Möglicherweise beabsichtigten die polnischen Behörden zunächst, die Deutschen durch Verhandlungen zu einer Wiederaufnahme der ausgewiesenen Personen in Deutschland zu bringen ${ }^{57}$. Die polnische Diplomatie war zu keinem Kompromiß bereit und mußte angesichts der Situation der Flüchtlinge auf polnischem Gebiet reagieren. Der Repräsentant des Joint befürch-

52 JDC, Folder 878, Bericht über einen Besuch in Zbanszyn, 13.-14. 11. 1938.

53 JDC, Folder 878, Summary Report on an Inspection Tour to Zbanszyn on July 17, 1939, by Mr. Leon Alter, Director of the JEAS, Warschau.

54 Zur Mäßigung des polnischen Antisemitismus in jener Zeit siehe die Aussage eines Betroffenen: „Die Bevölkerung war sehr hilfsbereit und hat auch Flüchtlinge aufgenommen, teilweise ohne Bezahlung und für geringe Vergütung. [...] Die Bevölkerung ist sehr deutschfeindlich. Nur in einer Wirtschaft war fast immer der deutsche Sender angestellt. Auch die polnischen Beamten waren anständig, aber böse darüber; daß kein polnisch gesprochen wurde." - YVA, 02/427, Die „Polenaktion“. - In einem Briefe Emmanuel Ringelblums, einer der Leiter des Joint-Komitees für Zbanszyn, an einen Betroffenen, der inzwischen nach Palästina emigriert war, heißt es: „Die Distanzierung unseres westlichen Nachbarn machte sich bei uns in einer vorläufigen Abschwächung des Antisemitismus bemerkbar. Auf jeden Fall ereignen sich derartige Phänomene, wie es sie in der Zeit gab, in der ich in Polen war, jetzt nicht mehr." - Brief E. Ringelblums an Arnon Fishman-Tamir bei: Mahler, Ringelblum Letters, S. $28 \mathrm{f}$.

55 JDC, Folder 878, Jews Exiled from Germany to Poland, December 1938.

56 Maurer, Abschiebung, S. 65.

57 Tomaszewsky, Zbanszyn, S. 228. 
tete, die polnische Regierung könnte die Ausgewiesenen in Konzentrationslagern sammeln ${ }^{58}$.

Schließlich entschied sich Polen, aus der entstandenen Situation den größtmöglichen Nutzen zu ziehen und die Ausgewiesenen in Zbanszyn zu instrumentalisieren, um das Problem der jüdischen Emigration - nach dem Scheitern der Evian-Konferenz - erneut auf die internationale Tagesordnung zu bringen. $\mathrm{Zu}$ diesem Zwecke übten polnische Diplomaten in Washington, Den Haag und London Druck auf die jeweiligen Regierungen aus, um eine Aufnahme der Flüchtlinge in diesen und anderen Ländern zu erreichen. Sollten die Forderungen der polnischen Regierung nicht erfüllt werden, erklärten sie, werde sie antisemitische Ausbrüche in allen Regionen Polens initiieren ${ }^{59}$. Parallel zu diesen Bemühungen auf diplomatischer Ebene begann die polnische Regierung, die Juden Polens für ihre Absichten einzuspannen ${ }^{60}$. Sie forderte von jüdischen Organisationen in Polen, Telegramme an jüdische Organisationen in aller Welt (American-Jewish Committee, Jewish-American Congress, Jerwish Board of Deputies in London, Alliance Israélite Universelle u. a.) zu senden und die irreführende Information zu verbreiten, Polen helfe den Ausgewiesenen und fordere, im Gegenzug die polnischen Juden in die Hilfsprogramme für deutsche Juden einzuschließen. Einige Tage später trug die polnische Regierung der jüdischen Führung in Polen auf, ein Jüdisches Komitee für Emigration und Kolonisierung zu errichten, dem Professor Schorr vorstehen sollte.

Die polnische Regierung wollte mit diesen Maßnahmen den Eindruck erwecken, die polnischen Juden identifizierten sich mit dem Evakuierungsprogramm der Regierung. Die jüdische Presse in Polen, der der Zwangscharakter der Maßnahmen verborgen geblieben war, trat mit heftiger Kritik hervor, was die Regierung jedoch nicht hinderte, derartige Aktivitäten fortzusetzen. Einige Tage später wurden Schorr und andere Vertreter von der polnischen Regierung vorgeladen, wo ihnen mitgeteilt wurde, sie hätten Filialen des Jüdischen Komitees für Emigration und Kolonisierung in ganz Polen aufzubauen und eine Landeskonferenz einzuberufen, die alle Juden in Polen aufrufen sollte, insgesamt drei Millionen Zloty für die Emigrationsmaßnahmen aufzubringen. Gleichzeitig gründete die Regierung eine nichtjüdische Organisation namens Freunde des Emigrationsplans nach Madagaskar. Darüber hinaus lud die polnische Regierung jüdische Führungspersönlichkeiten vor und drohte ihnen, wenn „der Preis, den Deutschland für die Brutalität gegen Juden erhalte, darin bestehe, daß Juden aus diesem Land entfernt werden, so bleibe Polen

58 JDC, Folder 878, Bericht Hellman, 10. 11. 1938. - Polnische Nachrichtenagenturen verbreiteten Gerüchte über die Bereitschaft der polnischen Regierung, polnische Juden aus Wien, die trotz des Gesetzes über die Erneuerung der Staatsangehörigkeit auf polnisches Gebiet gelangt waren, in dem Konzentrationslager Bereza Kartuska zu internieren; Bauer, Brother's Keeper, S. 244.

59 Brief Herbert von Dirksens (Ribbentrops Nachfolger als deutscher Botschafter in London) an Ernst Wörmann, den zweiten Mann in der deutschen Botschaft in London, London, 30. 11. 1938, bei: Milton, Expulsion, S. 189. - Die polnischen Versuche, dem Unterkomitee für Flüchtlingsfragen der Evian-Konferenz die Diskussion der Frage der aus Deutschland Abgeschobenen aufzuzwingen, war nicht erfolgreich: Maurer, Abschiebung, S. 67.

60 Die Informationen über die Aktivitäten der polnischen Regierung innerhalb Polens stützen sich auf den Joint-Bericht JDC, Folder 793, Situation of the Jews in Poland, 29. 11. 1938, sowie Bauer, Brother's Keeper, S. $247 \mathrm{ff}$. 
nichts anderes übrig, als zu ähnlichen Methoden im Hinblick auf die jüdische Emigration aus Polen zu greifen" 61 .

Die gleichen Absichten verfolgte die polnische Regierung mit ihrer Informationspolitik über die Ereignisse in Zbanszyn. Während es in den ersten Tagen der Presse verboten worden war, über die Lage in der Grenzstadt zu berichten, fiel kurz darauf in Übereinstimmung mit der neuen politischen Linie die Entscheidung, Zbanszyn zu Propagandazwecken zu instrumentalisieren. Polnische Journalisten wurden eingeladen, als Beobachter die Verhältnisse im Flüchtlingslager zu schildern, wobei sie vom polnischen Außenministerium auf einer eigens für nichtjüdische Journalisten eingerichteten Pressekonferenz angewiesen wurden, den Akzent in ihren Berichten auf das Emigrationsproblem in Polen zu setzen. Polen könne sich gegenüber der jüdischen Bevölkerung nicht mehr tolerant verhalten, wenn dies die Juden ermutige, in Polen zu verbleiben, während gleichzeitig Deutschland sich seiner jüdischen Bevölkerung mit internationaler Hilfe entledige, indem es Juden rücksichtslos behandele ${ }^{62}$. Wie schon in der Vergangenheit sollte die Diskussion um die Emigration gleichermaßen innen- wie außenpolitischen Zwecken der polnischen Regierung dienen. Emmanuel Ringelblum ${ }^{63}$, der sich im Auftrag des Joint in Zbanszyn aufhielt, war überzeugt, die polnische Regierung habe ihre Intention geändert, es gehe ihr nicht mehr um eine Machtdemonstration gegenüber Deutschland, sondern um „Propagandamittel gegen die Anhänger der Endecja“64.

Gleichzeitig weigerte sich Polen, Ansätze konstruktiven Handelns zu demonstrieren, um die Chancen der Emigration für polnische Juden aus Deutschland zu verbessern. Der polnische Botschafter in Deutschland, Lipski, informierte den polnischen Außenminister Beck, seit Mitte November 1938 würden nur noch selten polnische Pässe von Juden in Deutschland gestempelt und das Gesetz gegen die Erneuerung der Staatsbürgerschaft werde im März in Kraft treten ${ }^{65}$. Die Weigerung der polnischen Konsulate aber, die Pässe zu verlängern, schränkte die Einwanderungsmöglichkeiten in die Vereinigten Staaten ebenso ein wie die nach Palästina zu emigrieren ${ }^{66}$. Am 24. Januar 1939 unterzeichnete Polen ein Abkommen mit Deutschland, in dem es sich verpflichtete, den Ausgewiesenen, die zur Regelungen ihrer finanziellen Angelegenheiten nach Deutschland gefahren waren, die Rückkehr nach Polen zu gestatten und den in Deutschland zurückgebliebenen Familienangehörigen eine Zusammenführung mit ihren Verwandten in Polen zu ermöglichen. Deutschland seinerseits setzte die Ausweisung einzelner polnischer Juden über die grüne Grenze nach Polen fort ${ }^{67}$.

Es ist kaum verständlich, warum die polnische Regierung unter den beschriebenen Umständen die Abgeschobenen weiterhin in dem Flüchtlingslager in Zbanszyn

61 Ebenda.

62 Ebenda, sowie bei: JDC, Folder 793, Memorandum from Henrietta Buchmann.

$63 \mathrm{Zu}$ Ringelblums Tätigkeit als Historiker und politisch engagierter Mann siehe: Cohen, Emmanuel Ringelblum.

64 Brief Ringelblums an Raffael Mahler vom 6. 12. 1938, in: Mahler, Ringelblum Letters, S. 24.

65 Lipski, Doc. 126, Lipski an Beck, Berlin, 12. 11. 1938, S. 464.

66 AAN, Amb Rp w Berlinie, Akt Nr. 3043, Amerikanisches Generalkonsulat an das Generalkonsulat der Republik Polen in Berlin, 22.12. 1938; Palästina-Amt Berlin an das Konsulat der Republik Polen in Oppeln, 26. 1. 1939.

67 BAK, Der Reichsführer SS und Chef der Deutschen Polizei an verschiedene Stellen am 8. 5. 1939; Jonca, Niemiecko Polski, S. 118 f., Maurer, Abschiebung, S. 69. 
festhielt ${ }^{68}$. In dem Beharren auf dem Lager in Zbanszyn zeigten sich die Schwierigkeiten der polnischen Seite anzuerkennen, daß die Aktion zur Aufhebung der Staatsbürgerschaft ein Fehler gewesen war und kein politischer Gewinn aus diesem Unternehmen erzielt werden konnte. Mitte Juli 1939 hielten sich noch immer fast 3000 Flüchtlinge in dem Lager auf. Die Vertreter des Joint sahen diese Lage als unmittelbare Folge der politischen Verhältnisse in Polen und hegten keinerlei Hoffnung, die polnische Regierung werde sich zu einer Auflösung des Lagers bewegen lassen ${ }^{69}$. Am 31. August 1939 berichtete der Vertreter des Joint in Paris der Organisationsleitung in New York von einem Treffen mit Giterman, dem Leiter des Joint in Polen, der meinte, es habe keinen Sinn, sich wegen der Aufhebung der polnischen Staatsbürgerschaft an die polnischen Behörden zu wenden ${ }^{70}$. Die Aufhebung der Staatsbürgerschaft, so Giterman, sei keine administrative Maßnahme, sondern die Implementierung eines vom polnischen Sejm verabschiedeten Gesetzes und liege daher außerhalb der Entscheidungskompetenz der Beamten. Einen Tag später überfiel Deutschland die Republik Polen - der Zweite Weltkrieg hatte begonnen.

Die Versorgung der Ausgewiesenen in Polen mußte zum größten Teil von den jüdischen Gemeinden und Organisationen übernommen werden ${ }^{71}$. Zunächst brauchten die Flüchtlinge Lebensmittel und Getränke. Ausgewiesene, denen es gelang, die jüdischen Gemeinden in Posen, Krakau, Lodz und anderen Großstädten zu erreichen, konnten in vielen Fällen bei jüdischen Familien unterkommen und waren von dem Ausmaß der Solidarität und Herzlichkeit überrascht, die ihnen dort begegneten. Doch diese erste, spontane Hilfsbereitschaft reichte nicht aus, denn das Flüchtlingsproblem konnte nicht vor Ort gelöst werden, sondern erforderte eine langfristige Lösung. Die polnischen Behörden hegten keinerlei Absicht, den Aufenthalt der Flüchtlinge in Polen zu finanzieren. So verblieben die organisatorische Verantwortung und die finanzielle Belastung für die Integration der Flüchtlinge und die Versorgung der in Zbanszyn und Posen konzentrierten Abgeschobenen bei den jüdischen Organisationen und der jüdischen Öffentlichkeit. Aber auch hier wurde Beachtenswertes geleistet ${ }^{72}$. In ganz Polen wurden Hilfskomitees eingerichtet, die den Flüchtlingen aus Spenden von einheimischen Juden sowie aus Zusatzmitteln des Joint in allen Lebensbereichen kontinuierliche Unterstützung zukommen ließen ${ }^{73}$.

Die Position des Joint wurde rasch deutlich: Das Lager in Zbanszyn sollte nicht zur Dauereinrichtung werden, um nicht Modellcharakter für die polnischen Behör-

68 Tomaszewski meint, schon Ende November hätten die polnischen Behörden beschlossen, das Lager Zbanszyn schrittweise aufzulösen, belegt jedoch seine Behauptung nicht. Andere Angaben widerlegen diese Version: Tomaszweski, Zbanszyn, S. $228 \mathrm{f}$.

69 JDC, Folder 874, Status of Refugee Problems in Poland, 11. 7. 1939.

70 JDC, Folder 878, M.C. Troper (JDC Paris) an JDC New York, 31. 8. 1939.

71 Der freundliche Empfang, der den Flüchtlingen von jüdischer Seite bereitet wurde, fand seinen Niederschlag wiederholt in etlichen Zeugenaussagen: YVA, M-1/E-1903/1753; 02/486; 03/915; Brief Julius Rosenzweigs, in: Milton, The Expulsion (Anm. 8), S. 180; JDC, Folder 878, Eye witness report of Rescue Activities of JDC Polish-German Border, 18. 11. 1938.

72 Ringelblum beschrieb das Motiv für die große Hilfsbereitschaft mit den Worten: „Zbanszyn war ein harter moralischer Schlag gegen die jüdische Bevölkerung in Polen. Daher richteten sich die Blicke der jüdischen Massen auf Zbanszyn und die leidenden Juden." - Brief Ringelblums an R. Mahler vom 6. 12. 1938, in: Mahler, Ringelblum Letters, S. 25.

73 Zur Arbeit der Komitees siehe: Bauer, Brother's Keeper, S. $245 \mathrm{ff}$. 
den zu gewinnen ${ }^{74}$; die finanzielle Verantwortung für die Arbeit in Zbanszyn sollte im wesentlichen von jüdischen Einrichtungen in Polen übernommen werden ${ }^{75}$. Beide Positionen waren äußerst problematisch: Polen blieb nämlich beharrlich dabei, die Flüchtlinge in Zbanszyn zu belassen. Angesichts der schweren Winterbedingungen sah sich der Joint also gezwungen, die Verhältnisse der Flüchtlinge zu verbessern. Darüber hinaus waren sich die Leiter des Joint schon zu Beginn der Arbeit der Organisation in Zbanszyn der geringen Durchhaltekräfte des polnischen Judentums bewußt und fürchteten, je mehr die Not der Flüchtlinge zu einem permanenten Zustand wurde, desto geringer könnte die Opferbereitschaft werden ${ }^{76}$. Ringelblum, der sich im Lager aufhielt, beklagte die gewaltige Last, die der jüdischen Öffentlichkeit durch die Versorgung der Flüchtlinge auferlegt wurde:

„Ich frage mich, warum die ganze Welt schweigt. Unsere Staatsangehörigen, keine Ausländer, Besitzer gültiger Pässe, werden monatelang an der Grenze festgehalten. Ihre Versorgung wird der jüdischen Bevölkerung auferlegt. Das kostet zwölftausend Zloty am Tag. Wie lange können sie diese Kosten tragen? Deswegen werden tausende anderer Aktivitäten beeinträchtigt. Man könnte ruhig sagen, alle öffentliche Tätigkeit ist deswegen zum Stillstand gekommen." "77

Darüber hinaus verteilte sich die Last der Flüchtlingsversorgung nicht gleichmäßig auf die gesamte jüdische Bevölkerung in Polen. Im Abschlußbericht der Komiteearbeit vermerkt der Joint:

„Die allgemeine Erfahrung des Komitees hat gezeigt, daß die beste Reaktion von seiten des Mittelstandes gekommen ist. Dort hat man nicht nur ein außergewöhnliches Verständnis für das Problem gezeigt, sondern auch wirklich praktischen Enthusiasmus bei dem Versuch, das Problem zu lösen. Ein bedauernswertes Phänomen war die Gleichgültigkeit und mangelnde Großzügigkeit der wohlhabenderen Juden. Sie blieben anscheinend von dem schreienden Elend anderer unberührt, sie haben außerordentlich wenig beigetragen im Vergleich zu ihrem enormen Reichtum."

Die Leiter des Joint waren also mit der Hilfsbereitschaft der polnischen Juden keineswegs zufrieden. Um eine Erhöhung der eigenen Ausgaben für die Vertriebenen zu vermeiden, wandte sich die Organisation mit Spendenaufrufen an die Landsmannschaften der polnischen Juden in Amerika ${ }^{79}$. Weitere Gelder kamen von Organisationen polnischer Juden in England, Belgien, Holland, Frankreich und Schwe$\operatorname{den}^{80}$.

Abgesehen von den schweren Budgetproblemen, die die jüdische Wohlfahrtsarbeit in Polen stets begleitet hatten, behinderte die starke politische Splitterung des

74 Ebenda, S. 246.

75 Ebenda, S. $246 \mathrm{f}$.

76 JDC, Folder 878, Troper an Hyman, 1. 12. 1938.

77 Brief Ringelblums an Mahler am 3. 12. 1938, in: Mahler, Ringelblum Letters, S. 26.

78 JDC, Folder 172, J.C. Hyman an die Mitarbeiter und Mitglieder des Exekutivkomitees des JDC, 17. 2. 1939.

79 Das Joint-Distribution Komitee, Landsmannschaft Divisia, Chaim Weintraub (Sekretär), 28. 12. 1938; "Die aus Deutschland ausgesiedelten Drohobyczer und die Drohobyczer Landsleute“, 27. 12. 1938; 480 Männer als Familienoberhäupter und selbständige Frauen der ,Landsmannschaft Krakow' in Zbanszyn an Brüder und Schwestern aus Krakau, 14. 12. 1938; JDC, Folder 878, Buchman an Chaim Weintraub, 15. 12. 1938.

80 JDC, Folder 172, J.C. Hyman an die Mitarbeiter und Mitglieder des Exekutivkomitees des JDC, 17. 2. 1939. 
polnischen Judentums die Bemühungen um eine Lösung der Flüchtlingsfrage ${ }^{81}$. Im Dezember 1938, also einige Wochen nach der Ausweisung, wandten sich die Leiter des Hechalutz in Polen an die Verwaltung des Joint mit einem Vorschlag, der sofort hätte verwirklicht werden können, wenn die polnische Regierung eine Auflösung des Lagers genehmigt hätte ${ }^{82}$. Ein Teil der Ausgewiesenen hatte sich mitten in einem für die Einwanderung nach Palästina obligatorischen landwirtschaftlichen Ausbildungskurs [Hachscharab] befunden, als sie aus Deutschland abgeschoben wurden. Andere waren Mitglieder der Jugendbewegungen, die sie auf eine Hachscharah vorbereiten sollten. Der Hechalutz war daran interessiert, diesen Jugendlichen die Beendigung ihrer Hachscharah zu ermöglichen und sie somit für eine Emigration nach Palästina zu qualifizieren. Die Jugendlichen in Zbanszyn organisierten inzwischen Gruppen und wandten sich mit der Bitte um Unterstützung an den Hechalutz in Polen. Die Organisation konnte zwar einzelnen erfolgreich helfen, hatte jedoch nicht genügend eigene Mittel, um das Problem insgesamt ohne Subventionen des Joint zu lösen. Absicht des Hechalutz war es, diese Jugendlichen gleichberechtigt in die Gruppen polnischer Pioniere in etablierteren Hachscharot-Programmen zu integrieren und für sie neue Beschäftigungsmöglichkeiten zu schaffen. $\mathrm{Zu}$ diesem Zwecke wandte sich der Hechalutz in Polen mit der Bitte um eine Teilübernahme der Kosten an den Joint.

Dieses Programm nahm dann auch Gestalt an. Der Joint zeigte grundsätzliche Bereitschaft, suchte jedoch nach zusätzlichen Finanzquellen, um die Bürde der Kosten nicht allein tragen zu müssen ${ }^{83}$. In der Zwischenzeit wandte sich auch der Bund mit einem anderen Vorschlag an den Joint. Infolge eines Treffens zwischen dem Vertreter des Bundes, Henryk Erlich, dem Vertreter der polnischen Sozialistischen Partei, Artiscewski, und dem polnischen Wohlfahrtsminister Marian Zyndram Koscialkowski hatte der Bund die Zustimmung des Wohlfahrtsministers erlangt, er werde eine Freilassung aller Kinder und Jugendlichen unter 20 Jahren aus Zbanszyn zirka 1400 Menschen - erwirken, wenn der Bund sich verpflichte, daß die Kosten für ihre Versorgung nicht zu Lasten der polnischen Regierung gehen werden ${ }^{84}$. Der Bund wandte sich daher an den Joint mit der Bitte um eine finanzielle Beteiligung. Als Gerüchte von diesen Absprachen den zionistischen Aktivisten zur Kenntnis gelangten, reagierten sie höchst verärgert. Sie sahen darin einen Versuch des Bundes, der im Unterschied zu den Zionisten nicht an den Hilfskomitees beteiligt war, diese Komitees zu umgehen und dadurch Kinder und Jugendliche dem Zionismus zu entziehen und dem Bund zuzuführen ${ }^{85}$. Bernard Kahn, der Leiter des Joint in Europa, hatte den Sprengstoff, der in diesem Konflikt lag, sehr wohl erkannt und versuchte, die Gemüter zu beruhigen, indem er erklärte, daß der Joint nicht beabsichtige, die volle Verantwortung für die Kinder in Zbanszyn dem Bund zu übertragen ${ }^{86}$. Der

81 Das Fehlen einer zentralen Körperschaft des polnischen Judentums führte dazu, daß die Hauptlast der Flüchtlingsversorgung von den ohnehin verarmten Gemeinden getragen werden mußte. Melzer, No Way out, S. 126.

82 JDC, Folder 878, Giterman (JDC Warschau) an JDC Paris, 7. 12. 1938.

83 Ebenda; JDC, Folder 878, Tropper (Vorsitzender der europäischen Exekutive) an JDC New York, 16. 12. 1938.

84 JDC, Folder 878, Confidential Memorandum, JDC Paris, 12. 2. 1939.

85 JDC, Folder 878, Reiss, Hellman (Zionist Polen) an Kahn, 19. 2. 1939.

86 JDC, Folder 878, Henrietta K. Buchmann für J.C. Hyman an JDC Paris, 22. 3. 1939. 
Hechalutz war in diesen Auseinandersetzungen in einer günstigeren Position und konnte in Zusammenarbeit mit dem Joint seine Hachscharot für einige Hundert der ausgewiesenen Kinder fortsetzen. Ihnen wurde dadurch eine Ausbildung ermöglicht, und mehr als hundert jungen Männern und Frauen gelang so die Einwanderung nach Palästina noch vor Kriegsausbruch ${ }^{87}$.

Mit jedem Monat verringerten sich die finanziellen Mittel der einheimischen Gemeinden, und die Probleme verschärften sich Mitte Mai, als in Polen Anleihen für die Luftabwehr durchgeführt wurden. Jetzt wurde es polnischen Juden verboten, parallel dazu Spendenaktionen durchzuführen ${ }^{88}$. Viele lokale Komitees mußten ihre Arbeit einstellen, und der Joint sah sich gezwungen, weitere Gelder zu investieren, um eine Wiederbelebung der Arbeit zu ermöglichen ${ }^{89}$. In einem Brief an den Finanzverwalter des Joint, Josef Hyman, thematisierte Morris Troper, Nachfolger Bernard Kahns, die Grenzen der Flüchtlingsarbeit des Joint:

„Von Zeit zu Zeit habe ich versucht, die Vertreter des polnischen Judentums darauf hinzuweisen, daß der Joint kein Monopol oder Patentrecht auf Polen hat und daß sie Unterstützung aus anderen Quellen suchen sollten und müßten. Es wäre zweifellos ein irreführendes Verhalten, wenn man sie in dem Glauben beließe, daß wir alleine die Verantwortung für derartig schwierige Probleme tragen könnten, mit denen sie heute konfrontiert werden ... Unglücklicherweise gibt es augenblicklich kein anderes Land als Amerika, in dem für die polnischen Juden grundsätzlich etwas getan wird. Diese Situation muß offensichtlich verändert werden, und ich glaube fest, daß die Errichtung eines Zentralkomitees in Polen das beste Mittel ist, um dieses Ergebnis zu erreichen." 90

Dazu kam es allerdings bis zum Kriegsausbruch nicht mehr.

\section{In Deutschland „zurückgeblieben“: Von Mitgliedern jüdischer Gemeinden zu Bürgern eines feindlichen Staates}

Während polnische Juden sich mit den Problemen der nach Polen ausgewiesenen Glaubensbrüder und -schwestern abmühten, waren in Deutschland mehr als 11500 polnische Juden zurückgeblieben ${ }^{91}$. Es waren im wesentlichen diejenigen, die der zweitägigen Massenausweisungsaktion im Oktober entkommen waren, sowie Frauen, Kinder und andere Ausgewiesene, deren Abschiebung nicht durchgeführt wurde, oder Personen, denen die Rückkehr nach Deutschland zur Regelung ihrer finanziellen Angelegenheiten genehmigt worden war. Die deutsche Politik zielte in jener Zeit auf eine Fortsetzung der Ausweisung kleinerer Gruppen polnischer Juden über die Grenze ${ }^{92}$ sowie auf die Kontrolle der Aktivitäten der temporären

87 Siehe die vorangegangene Darstellung zu den Hachscharot.

88 Melzer, No Way out, S. 125.

89 JDC, Folder 878, Morris C.Troper an Hyman, 1. 7. 1939.

90 Ebenda.

91 Adelson, Jewish Polish Citizens, S. 107; Adler zählt dagegen 10000 polnische und 13000 staatenlose Juden: Adler, Der verwaltete Mensch, S. 92.

92 Fritz Berger vom Palästina-Amt in Berlin wurde von der Berliner Polizei vorgeladen, um Informationen über illegale Grenzübergänge zu geben, die das Berliner Amt nutzte, um polnische Juden nach Polen zu bringen. Damit war keine Strafverfolgung des Palästina-Amtes intendiert, vielmehr wollte die SS diese Grenzübergänge ebenfalls zur Abschiebung nutzen. YVA, 01/4, Fritz Berger, Polenaktion 1938. 
Rückkehrer und bemühte sich um die genaue Registrierung der polnischen Juden und der Staatenlosen polnischer Herkunft ${ }^{93}$. Die jüdischen Gemeinden setzten die Unterstützung der Familien ausgewiesener Personen fort und halfen denjenigen, die zur Auflösung ihrer Unternehmen nach Deutschland zurückgekommen waren. Als der Juni näherrückte und damit die Frist, die der deutsch-polnische Vertrag für die Regelung der Angelegenheiten der Ausgewiesenen festgesetzt hatte, schaltete sich die Reichsvereinigung der Juden in Deutschland in die Bemühungen um weitere Aufenthaltsgenehmigungen ein und versuchte, den polnischen Juden zu Verlängerungen ihrer Aufenthaltsgenehmigungen in Deutschland zu verhelfen ${ }^{94}$.

Der Krieg erschütterte dann aber auch die Beziehungen zwischen dem deutschjüdischen Establishment und den wenigen Tausend in Deutschland verbliebenen polnischen Juden bis in die Fundamente. Am 8. September 1939 gab die Gestapo den Befehl, alle polnisch-jüdischen Männer über 16 Jahre zu verhaften, ihre Angehörigen zu registrieren und sie zu verpflichten, sich dreimal täglich auf einer Polizeistation zu melden ${ }^{95}$. Mit Kriegsausbruch konnten polnische Juden als Bürger einer verfeindeten Nation interniert werden, und es bestand keinerlei Notwendigkeit mehr, sie mit besonderer Rücksicht zu behandeln. Über das Schicksal der polnischen Juden in Deutschland nach Ausbruch des Weltkrieges gibt es nur wenige Zeugnisse. Aus dem ehemaligen Österreich wurden kurz danach 3000 polnische Juden nach Polen deportiert, wobei sie von der jüdischen Gemeinde zu Wien mit Nahrungsmitteln und Arbeitsgeräten ausgestattet wurden, damit sie sich auf polnischem Gebiet ein Hüttendorf errichten konnten ${ }^{96}$. Polnische Juden in Deutschland wurden in der Regel in Buchenwald interniert. Wer sich in Berlin aufhielt, kam in das Gefängnis Oranienburg und wurde von dort ins Konzentrationslager Sachsenhausen deportiert. Die Dokumente, die zu einer Rekonstruktion der Geschichte jener Juden beitragen könnten, sind nicht mehr vorhanden, viele sind sicherlich vernichtet worden. Die Akten der Reichsvereinigung, der Gemeinde zu Berlin, des Hilfsvereins oder der Organisation polnischer Juden sowie des Palästina-Amtes in Berlin - also der Organisationen, die sich um die verbliebenen polnischen Juden kümmerten - liegen für das Jahr 1939 nicht vor. Praktisch existieren gegenwärtig nur Aussagen zweier zentraler Zeugen: die Aussagen eines Aktivisten des Verbandes polnischer Juden in Berlin, namens Ortner, sowie die Berichte Recha Freiers, die im Jugendwohlfahrtssystem der Berliner Gemeinde eine führende Rolle gespielt und die Jugend-Aliyah gegründet hatte. Die Berichte beider Personen wurden in Israel lange nach der Shoah in verschiedenen Zusammenhängen aufgenommen. Sie stimmen in den wesentlichen Aussagen überein und werden von den wenigen vorhandenen Dokumenten bestätigt ${ }^{97}$. Demnach wurden im Juni 1939 alle deutschen

93 BAK, Sammlung Schumacher 240 II 1, Geheime Staatspolizei, Staatspolizeistelle Regensburg an Landräte, Staatskommissare ..., 10. 6. 1939; R 58/459, Reichsführer SS und Chef der Deutschen Polizei an verschiedene Stellen, 8. 5. 1939.

94 CAHJP, Inv. 6528 (133), Reichsvertretung der Juden in Deutschland, Abteilung Wanderung, an die jüdischen Gemeinden, Landes- und Provinzialverbände ..., 16. 6. 1939.

95 BLHA, Pr. Br. Rep. 6 B Kreisverwaltung Beerkow-Shorkow 834, Geheime Staatspolizei, Staatspolizeistelle Köln am 8. 9. 1939, Geheime Staatspolizei, Staatspolizeistelle Potsdam an Landräte, Oberbürgermeister ..., 8. 9. 1939.

96 Lageberichte des SD, Bericht zur innenpolitischen Lage Nr. 8, 25. 10. 1939, Bd. 2, S. 391.

97 IfCJ, 26/1, Aussage Ortner; YVA, 0-33/85, Aussage von Recha Freier. 
und staatenlosen Juden in Deutschland zwangsweise zu Mitgliedern der Reichsvereinigung der Juden in Deutschland, der Nachfolgeorganisation der Reichsvertretung der deutschen Juden, erklärt ${ }^{98}$. Die polnischen Juden waren fortan jedoch nicht mehr in der Reichsvereinigung eingeschlossen und konnten nur noch von seiten des Verbandes polnischer Juden unterstützt werden. Die Arbeit dieser Organisation wurde bei Kriegsausbruch zunächst nicht verboten. Als es in der ersten Septemberwoche zur Internierung aller polnisch-jüdischen Männer kam, wurden die Mitarbeiter der Organisation von der Haft verschont. Nach Ortners Aussage zeigten sich die deutschen Behörden an einer zentralen Körperschaft interessiert, die die Angelegenheiten der polnischen Juden regelte. Nach seiner und Recha Freiers Aussage war es Dr. Paul Eppstein vom Vorstand der Emigrationsabteilung der Reichsvereinigung, der der weiteren Existenz dieser Organisation eher abgeneigt war. Eppstein fürchtete, die jüdischen Angelegenheiten könnten mit den Problemen der Staatsangehörigen eines Deutschland feindlich gesonnenen Staates gleichgesetzt werden. Seine wiederholten Nachfragen bei den deutschen Behörden nach der Legalität der Organisation polnischer Juden veranlaßten die Gestapo letztlich, die Organisation am 19. September aufzulösen. Fortan existierte keine Körperschaft, die die Verantwortung für die Internierten und ihre Familien übernahm. Die Reichsvereinigung bestand auf ihrer Weigerung, einem ehemaligen Mitarbeiter des Verbandes polnischer Juden entsprechende Rechte zu übertragen und ihn als offiziellen Vertreter mit den Behörden in Kontakt treten zu lassen. Alle anderen jüdischen Organisationen waren ausnahmslos der Reichsvereinigung unterstellt und weigerten sich daher ebenfalls, die polnischen Juden zu betreuen. Diese Umstände erwiesen sich als besonders tragisch, als zwei Wochen nach der Internierung, am Vorabend des Laubhüttenfestes, Informationen über den Tod vieler Internierter eintrafen. Die zirka 500 von derartigen Nachrichten betroffenen Frauen versammelten sich zu einer Demonstration vor dem Gebäude der jüdischen Gemeinde zu Berlin und unterbrachen den Festgottesdienst in der Synagoge. Die Demonstration schreckte die jüdischen Verantwortungsträger auf, da über die deutschen Juden ein Versammlungsverbot verhängt worden war. Der Vorsitzende der Berliner Gemeinde, Heinrich Stahl, war zwar grundsätzlich durchaus bereit, den Frauen und ihren Familien zu helfen, deren wirtschaftliche Situation sich in der Zwischenzeit aufgrund der Abwesenheit des Ehepartners verschlechtert hatte, doch auch ihm waren die Hände gebunden: Die Gemeinde zu Berlin war wie alle jüdischen Organisationen der Reichsvereinigung unterstellt, die, wie erwähnt, Hilfe für polnische Juden verweigerte. Nur unter dem Druck der Demonstrantion war Eppstein bereit, es dem Mitarbeiter des Verbandes polnischer Juden, Awerbuch, zu ermöglichen, die Angelegenheiten polnischer Juden vor den Behörden zu vertreten ${ }^{99}$.

Neben der wirtschaftlichen Hilfe für die Familien der Ausgewiesenen stand die Sorge um die Internierten. Jeden Tag kamen neue Meldungen von Todesfällen. Um die Freilassung von Internierten zu erwirken, war es von zentraler Bedeutung, die

98 Gegenüber der Behauptung, die Reichsvereinigung sei eine von seiten der Gestapo eingesetzte Organisation gewesen, betonen Kulka und Hildesheimer, daß es sich um eine freiwillige aus Eigeninitiative gegründete Körperschaft handelte, die unter völlig neuen Bedingungen die Aufgaben der Reichsvertretung fortsetzte: Kulka, Reichsvereinigung; Ders., Dokumente, Bd. 1, S. 27, 30, 441-446; Hildesheimer, Jüdische Selbstverwaltung, S. 63 ff., 77, $80 \mathrm{ff}$.

99 Folgende Darstellung stützt sich auf die Aussagen von Ortner und Freier. 
sofortige Auswanderung aus Deutschland zu ermöglichen. Praktisch genügte ein Zertifikat für eine Aliyah Beth, also eine illegale Einwanderung nach Palästina, um die deutschen Behörden zufriedenzustellen. Für eine Freilassung begnügte sich die SS mit Emigrationsbelegen jeglicher Art ${ }^{100}$. Der späteren Aussage eines der Mitarbeiter des Palästina-Amtes zufolge reichten äußerst einfache Belege aus, um die Befreiung vieler der Internierten zu erwirken: ein Schreiben des Hechalutz, ,in dem bescheinigt wurde, daß der Verhaftete gewillt sei auf Hachscbarab zu gehen"101. Das Palästina-Amt in Berlin konnte hier also eine zentrale Rolle spielen, obwohl es scheint, als ob es auf derartige Aufgaben nicht vorbereitet war ${ }^{102}$. Fritz Berger von der Leitung des Palästina-Amtes sagte viele Jahre später, daß am Ende des Zionistenkongresses in Genf, kurze Zeit nach Kriegsausbruch, entschieden worden sei, wer von den deutschen Delegierten nach Deutschland zurückkehren und wer nach Palästina einwandern sollte:

„Der maßgebende Gesichtspunkt war, ob die ausgeübte Art der Tätigkeit in Deutschland noch möglich und sinnvoll war. So wurde entschieden, daß Eppstein (Beziehung zur Gestapo) und Conrad Cohn (Wohlfahrtspflege) [...] nach Deutschland zurückzufahren hätten, während z.B. ich draußen bleiben sollte, weil alle polnischen Staatsangehörigen am Tage des Kriegsausbruchs in Konzentrationslager verbracht worden waren und mein Hauptarbeitsgebiet (Polenrettung) damit sinnlos geworden war." 103

Warum die Zionisten die Dringlichkeit der Hilfe für polnische Juden in Deutschland nicht verstanden und warum sie meinten, es bestünde keine Möglichkeit der Hilfe mehr, ist nicht klar.

Die Initiative zur Betreuung der Internierten hatte Recha Freier ergriffen. In einem ihr von der Gemeinde zur Verfügung gestellten Büro begann sie, dreimal in der Woche Sprechstunden abzuhalten und Informationen zu sammeln, die ihr von Müttern und Frauen der Inhaftierten, über Verwandte und über berufliche Chancen in Palästina mitgeteilt wurden. Das Palästina-Amt plante zwar zu jener Zeit für Mitte November 1939 eine illegale Einwanderungsaktion, doch bemühte sich Recha Freier mit wenig Erfolg, Einwanderungszertifikate für die Ausgewiesenen zu erhalten. Der Mitarbeiter des Palästina-Amtes Max Zimels beschreibt die Schwierigkeiten, die einer Ausgabe der Einwanderungszertifikate an Verhaftete im Wege standen:

100 StA Dresden, Ministerium des Innern Nr. 11678, Der Reichsführer SS an den Reichsstatthalter in Sachsen - Landesregierung - Ministerium des Innern in Dresden am 17.11. 1939; Der Polizeipräsident zu Chemnitz an den Sächsischen Minister des Innern zu Dresden, 16. 12. 1939.

101 YVA, 0-1/263, Max Zimels, In Berlin von Ende Dezember 1938 bis Anfang September 1939.

102 Rut Zariz widmet sich in ihrer Studie der Arbeit des Palästina-Amtes in Berlin den Ausgewiesenen, bezieht jedoch eine ausweichende Position im Hinblick auf die Qualität der Arbeit und die internen Spannungen: Zariz, Migration of German Jews, $59 \mathrm{ff}$.

103 YVA, 01/272, „Die Verhandlungen von Fritz Berger mit den nationalsozialistischen Behörden wegen zeitweiliger Rückkehr der am 28. Oktober 1938 ausgetriebenen polnischen Staatsangehörigen". Eine dritte, sehr kurze Aussage, die beide vorangehenden Aussagen stützt, stammt von Anni Nieder, die in der „Organisation polnischer Juden“ in Berlin und nach deren Auflösung im Palästina-Amt gearbeitet hat: YVA, 01/15, Polenaktion, Bericht von Anni Nieder. 
„Wir wurden damit vor eine schwere Entscheidung gestellt. Wir übernahmen mit der Bescheinigung die Verantwortung, den Betreffenden auch auf Auslandshachscharah oder nach Palästina zu schicken, ohne daß wir uns überzeugen konnten, ob er hierfür geeignet war. Wir hatten ja nicht einmal die Möglichkeit, die Betreffenden, die in Haft waren, persönlich zu sprechen, eine Ablehnung in gewissen Fällen war grausam, aber unerläßlich." 104

Nachdem Recha Freier nur 30 Zertifikate für die illegale Einwanderung nach Palästina Ende November hatte erwirken können, geriet sie in heftige Auseinandersetzungen über den Anteil der Ausgewiesenen an der für März-April 1940 geplanten Aliyah Beth. Ihr Antrag wurde abgewiesen. Nach ihren Worten erklärte ihr der Leiter des Palästina-Amtes, Franz Leon, die zionistische Bewegung bestünde nicht, um caritative Aufgaben zu erfüllen ${ }^{105}$. Auch Paul Eppstein wandte sich gegen Freier. Er schlug vor, sich an die deutschen Behörden zu wenden und um die Verlegung der jüdischen Internierten von Sachsenhausen nach Lublin zu bitten. Eppsteins Sorge, so Freier, sei es gewesen, die Gestapo könnte auf den Gedanken kommen, auch deutsche Juden zu inhaftieren, um ihre Emigration einzuleiten, wenn sie erst erkannt habe, daß den polnischen Internierten eine Auswanderung nach Palästina ermöglicht werde. Die Komiteemitglieder wiesen zwar Eppsteins Vorschlag zurück, entschieden jedoch mit großer Mehrheit, den Internierten keine gesonderten Einwanderungszertifikate zur Verfügung zu stellen. Eine weitere Intervention Eppsteins bei den deutschen Behörden führte dazu, daß diese erklärten, fortan würden keine Internierten im wehrfähigen Alter (ab Jahrgang 1900) aus der Haft entlassen, wenn das Ziel ihrer Auswanderung ein feindliches Land sei-darunter fiel auch $\mathrm{Pa}$ lästina.

Recha Freier übte im Verlauf des Monats Dezember verstärkten Druck auf das Palästina-Amt in Berlin aus und konnte 75 weitere Einwanderungszertifikate erwirken. Auf Umwegen gelang es ihr, noch einige andere Zertifikate zu erhalten; sie mußte jedoch bitter enttäuscht feststellen, daß die Internierten trotz der Vorlage der Zertifikate nicht aus der Haft entlassen wurden. Im nachhinein wurde ihr bekannt, daß auf Initiative der Gestapo beim Palästina-Amt angefragt worden war, ob die Träger der Zertifikate auch tatsächlich nach Palästina auswanderten. Das Palästina-Amt hatte eine eindeutige Antwort auf diese Frage abgelehnt ${ }^{106}$. Freiers Verzweiflung angesichts der Reaktion des Palästina-Amtes zeigt auf die tiefe Kluft zwischen ihrer Position und der Auffassung des Palästina-Amtes von zionistischer Arbeit:

„Was riskierte das Palästina-Amt, wenn es im Rahmen der Frage geblieben wäre und eine bejahende Antwort gegeben hätte? Für eine nicht vollzogene Auswanderung hafteten allein die Antragsteller. Sie konnten schlimmstenfalls erneut in ein Lager kommen, während der mehrmonatigen Freiheit konnten sie aber - falls die Alijah Beth nicht rechtzeitig zustande käme -

104 YVA, 0-1/263, Max Zimels, In Berlin von Ende Dezember 1938 bis Anfang September 1939.

105 Zariz, Migration of German Jews, S. 62. Zariz meint, die Reichsvereinigung in Berlin habe in diesem Konflikt der Priorität zwischen zwei Ansätzen geschwankt: daß einerseits Familien polnischer Herkunft bevorzugt behandelt werden müßten, da sie in größerer Gefahr schwebten, oder andererseits, daß Kandidaten für eine Auswanderung aufgrund zionistischer Maßstäbe aus der Vorkriegszeit ausgewählt werden müßten.

106 Freiers Bericht wird von der Aussage Anni Nieders gestützt: YVA, 0-1/15, Polenaktion, Bericht von Anni Nieder. 
eine der illegalen Auswanderungsmöglichkeiten in ein Zwischenland (Ungarn, Jugoslawien usw.) benützen. Das Palästina-Amt übernahm für die Internierten ebenso wenig wie für andere Auswanderer zur Alijah Beth irgend eine Garantie den Behörden gegenüber. "107

Indem sie den Legalismus der jüdischen Führung in Deutschland hinterfragte, berührte Freier hier eine Schlüsselfrage. Ihre Sicht der Verhältnisse wird trotz der defizitären Dokumentation jener Zeit von Aussagen anderer Beteiligter und durch einige wenige NS-Dokumente gestützt, die u. a. ihre Vermutung von der anfänglichen Bereitschaft der nationalsozialistischen Behörden, Teilnehmer an einer Aliyab Beth aus der Haft zu entlassen, bestätigen. Richtig ist, daß die Reichsvereinigung und die ihr unterstellten jüdischen Körperschaften ihre Arbeit in strenger Anlehnung an die gesetzlichen Vorschriften durchführten ${ }^{108}$. Artur Prinz, ein Mitarbeiter des Hilfsvereins, der als Kontaktmann zur Gestapo in Emigrationsfragen fungierte, berichtete allerdings von einer Änderung der Haltung der mit Emigrationsfragen befaßten jüdischen Stellen nach der Reichspogromnacht ${ }^{109}$. Während bis dahin die jüdischen Institutionen in Kooperation mit den staatlichen deutschen Auswanderungsämtern darauf geachtet hätten, daß die Emigration von Juden völlig legal und mit fehlerlosen Papieren verlief, habe sich die Politik infolge der Massenverhaftungen während der Reichspogromnacht radikal geändert. Die geregelte Emigrationspolitik sei von dem Gedanken, jeder müsse sein eigenes Leben retten, verdrängt worden. Die unter deutschen Juden besonders starke Tendenz zur Gesetzlichkeit sei von dem Prinzip abgelöst worden, nichts sei wichtiger als die Rettung von Menschenleben ${ }^{110}$. Dies traf auf die mit Unterstützung der Gestapo vollzogene Fälschung von Dokumenten fremder Staaten zu. Im Hinblick auf die Kontakte mit der Gestapo selbst dürfen wir wohl davon ausgehen, daß die jüdischen Institutionen weiterhin ausschließlich im Rahmen der existierenden gesetzlichen Vorschriften arbeiteten. Der Legalismus der Mitarbeiter des Palästina-Amtes in Berlin fügte sich also durchaus in die herkömmliche Strategie ein, die Emigration auf jedem möglichen Wege zu fördern - auch mit Hilfe von gefälschten Dokumenten und dem Verschweigen von Informationen.

Im Januar 1940 erörterte die Reichsvereinigung das Schicksal der polnisch-jüdischen Internierten in Sachsenhausen. Otto Hirsch, administrativer Leiter der Reichsvereinigung, schlug vor, „der zuständigen Behörde die Frage der Haftentlassung bei Unterbringung dieser Personen bei Angehörigen im Gebiet des damals polnischen Staates oder des Generalgouvernements vorzutragen"111. Die Motive, die Hirsch dazu veranlaßten, auf Eppsteins einige Wochen vorher gemachten Vorschlag zurückzugreifen, bleiben im dunkeln. Sollte er angenommen haben, die harten Bedingungen der Besatzung in Polen seien besser als die einer Haft in einem

107 Aussage von Recha Freier, S. 180.

108 Kwiet/Erschwege, Selbstbehauptung. - Kochavi, der Recha Freier und ihre Arbeit behandelte, erkennt eine historische Rechtfertigung für die legalistische Auffassung, die er als "Strategie der Defensive" bezeichnet, und zwar sowohl im Hinblick auf die Gefahren, die sich jeder aussetzte, der versuchte, die SS zu täuschen, als auch im Hinblick auf die Effektivität der illegalen Einwanderung: Kochavi, Deutsche Juden.

109 Prinz, Role of the Gestapo.

110 Ebenda. - Auch Kwiet geht davon aus, daß infolge des Novemberpogroms die jüdischen Institutionen die Emigration um jeden Preis unterstützt hätten, auch wenn diese den legalen Rahmen überschritt: Kwiet, To leave, S. 145.

111 RV, Protokoll der Vorstandssitzung der Reichsvereinigung, 21. 1. 1940. 
Konzentrationslager mit unklaren Verhältnissen, die täglich zu vielen Opfern führten? Hirsch wird wohl nichts von den schrecklichen Lebensbedingungen, von Hunger und Krankheiten gewußt haben, denen diejenigen Ausgewiesenen ausgesetzt waren, die in die von Deutschland besetzten Gebiete Polens gelangt waren ${ }^{112}$. Am 5. Februar 1940 erließ die SS den Befehl, polnische und staatenlose Juden aus den Konzentrationslagern und Haftanstalten in Deutschland ins sogenannte "Generalgouvernement" zu deportieren ${ }^{113}$. Hirsch ahnte nicht, was Juden, die zu jener Zeit nach Polen deportiert wurden, erwartete. Sein Vorschlag ist ein Beweis dafür, daß die Reichsvereinigung jegliche Hoffnung aufgegeben hatte, das Problem der polnischen Juden in Deutschland im Rahmen der eigenen Möglichkeiten zu lösen. Die Probleme der polnischen Juden wurden hinfort ausgeklammert. Einen Monat nach Hirschs Vorschlag und wenige Tage, nachdem die SS die Deportation der polnischen Juden ins Generalgouvernement angeordnet hatte, wurden 1200 deutsche Juden aus Stettin in den Distrikt Lublin deportiert ${ }^{114}$. Dieses Mal versuchte die Reichsvereinigung, ihre Rückkehr nach Deutschland zu erwirken ${ }^{115}$, denn es handelte sich um deutsche Juden.

112 Reitlinger, Endlösung, S. $45 \mathrm{ff}$.

113 StA Hamburg, Senatskommission für die Reichs- und Auswärtigen Angelegenheiten II, Signatur VI B 1 Fak. 49, Der Reichsführer SS und Chef der Deutschen Polizei an verschiedene Stellen, 5. 2. 1940.

114 Adler, Der verwaltete Mensch, S. 140f.; Reitlinger, Endlösung, S. 51 ff. - Die Korrespondenzen zwischen den Deportierten aus Stettin und ihren in Deutschland verbliebenen Verwandten sind gesammelt bei: Rosenfeld/Luckner, Piaski.

115 Adler, Der verwaltete Mensch, S. 142; Kulka, Reichsvereinigung, S. 54; Kwiet, To leave, S. 145. 